\title{
A Class of Double Integrals Involving Gaussian and Trigonometric Factors
}

\author{
D. M. Fradkin \\ Department of Physics, Wayne State University, Detroit, Michigan 48202* \\ April 16, 1979
}

\begin{abstract}
The five parameter double integral $\int_{0}^{\infty} d y \exp \left(-p^{2} y^{2}\right) \sin (\beta y+\theta)$ times $\int_{0}^{y} d x \exp \left(-x^{2}\right) \cos (\epsilon \beta x+\phi)$ is evaluated in terms of Fourier transforms of $\exp \left(-x^{2}\right) \operatorname{erfc}(\alpha x)$. Some new expressions for these transforms are obtained.
\end{abstract}

Key words: Definite integrals; double integrals; error functions; Fourier transforms; radiation reaction.

\section{Introduction}

The five parameter definite double integral considered in this paper is a generalization of that encountered in describing the radiation reaction effects on a charged particle swept over by a single plane laser pulse. The electric field for such a pulse can be described by the function

$$
\mathbf{E}(u)=\mathbf{E}^{\circ} \exp \left(-u^{2} / D^{2}\right) \sin (u / \lambda)
$$

where $u=c t-\hat{k} \cdot \mathbf{x}, \hat{k}$ being the direction normal to the plane wave.

The integral encountered is

$$
\mathbf{M}=\int_{-\infty}^{\infty} d \xi \mathbf{E}(\xi) \int_{-\infty}^{\xi} \mathbf{E}(\eta) \cdot \mathbf{E}(\eta) d \eta
$$

which in the notation of this paper may be expressed as

$$
\mathbf{M}=1 / 2 D^{2}\left(\mathbf{E}^{\circ} \cdot \mathbf{E}^{\circ}\right) \mathbf{E}^{\circ}[I(1 / \sqrt{2}, \sqrt{2} \kappa, 0 ; 0,0)-I(1 / \sqrt{2}, \sqrt{2} \kappa, 0 ; 2,0)]
$$

and evaluated to yield ${ }^{1}$

$$
\mathbf{M}=1 / 2 D^{2}\left(\mathbf{E}^{\circ} \cdot \mathbf{E}^{\circ}\right) \mathbf{E}^{\circ}\left[\exp \left(-\kappa^{2}\right) \operatorname{erfi}(\sqrt{2} \kappa / \sqrt{3})-1 / 2 \exp \left(-3 \kappa^{2}\right) \operatorname{erfi}(2 \sqrt{2} \kappa / \sqrt{3})\right]
$$

where $\kappa=D /(2 \lambda)$.

The five parameter definite double integral containing Gaussian and trigometric factors, discussed in this paper is

$$
I(p, \beta, \theta ; \epsilon, \phi) \equiv \int_{0}^{\infty} d y \exp \left(-p^{2} y^{2}\right) \sin (\beta y+\theta) \int_{0}^{y} d x \exp \left(-x^{2}\right) \cos (\epsilon \beta x+\phi)
$$

where $p>0, \beta \geq 0$, and $\epsilon \geq 0$. A somewhat related indefinite double integral, containing only Gaussian factors, has previously been discussed by Rosser [3]. It will be shown that $I(p, \beta, \theta ; \epsilon, \phi)$ may be evaluated in terms of Fourier transforms of Gaussian weighted erfc functions. The relevant Fourier sine transforms are

\footnotetext{
* Invited paper.

${ }^{1}$ The normalization adopted for the erf, erfe, and erfi functions in this paper is the same as that employed in references [1] and [2]. Figures in brackets indicate literature references at the end of this paper.
} 
available in closed form. Closed form expressions of such Fourier cosine transforms also exist for special cases, and integral forms convenient for numerical evaluation may be developed for the general case. The necessary Fourier transforms are discussed in the next section.

\section{Fourier Transforms of $\exp \left(-\mathbf{x}^{2}\right) \operatorname{erfc}(\alpha \mathbf{x})$}

The Fourier sine transform, for $\alpha \geq 0$,

$$
F_{s}(2 \beta, \alpha) \equiv \int_{0}^{\infty} \sin (2 \beta x) \exp \left(-x^{2}\right) \operatorname{erfc}(\alpha x) d x
$$

may be obtained from the formula given by Ng and Geller [4], p. 155, 3.5 (33), which may be put in the form

$$
F_{s}(2 \beta, \alpha)=\frac{\sqrt{\pi}}{2} \exp \left(-\beta^{2}\right)\left\{\operatorname{erfi}[\beta]-\operatorname{erfi}\left[\beta \alpha\left(\alpha^{2}+1\right)^{-1 / 2}\right\}\right. \text {. }
$$

For $\alpha<0$, one may use the relation

$$
\operatorname{erfc}(-x)=2 \operatorname{erfc}(0)-\operatorname{erfc}(x)
$$

to obtain the Fourier sine transform connection

$$
F_{s}(2 \beta,-|\alpha|)=2 F_{s}(2 \beta, 0)-F_{s}(2 \beta,|\alpha|) .
$$

The analogous Fourier consine transform, for $\alpha \geq 0$, is defined here as

$$
F_{c}(2 \beta, \alpha) \equiv \int_{0}^{\infty} \cos (2 \beta x) \exp \left(-x^{2}\right) \operatorname{erfc}(\alpha x) d x .
$$

Two closed form special cases of this are

$$
F_{c}(2 \beta, 0)=(\sqrt{\pi} / 2) \exp \left(-\beta^{2}\right)
$$

and

$$
F_{c}(0, \alpha)=(\pi)^{-1 / 2} \arctan \left(\alpha^{-1}\right) .
$$

This latter equation follows from reference [1], p. 7, 4.3(2). We shall demonstrate that for the general case, the Fourier cosine transform may be written as

$$
F_{c}(2 \beta, \alpha)=\exp \left(-\beta^{2}\right)\left[(\pi)^{-1 / 2} \arctan \left(\alpha^{-1}\right)+\alpha \int_{0}^{\beta\left(1+\alpha^{2}\right)^{-1 / 2}} \exp \left(\alpha^{2} x^{2}\right) \operatorname{erfi}(x) d x\right]
$$

or alternatively

$$
F_{c}(2 \beta, \alpha)=\alpha(\pi)^{-1 / 2} \exp \left[-\beta^{2}\left(1+\alpha^{2}\right)^{-1}\right] \text { times } \int_{0}^{1}\left[z^{2}+\alpha^{2}\right)^{-1} \exp \left[\beta^{2} z^{2}\left(1+\alpha^{2}\right)^{-1}\right] d z .
$$

These forms may be convenient for numerical evaluation when closed form expressions are not feasible. For $\alpha=1$, the integral in eq (9) may be evaluated (see reference [1], p. 7, 4.3(1)) to give the additional closed form special case

$$
F_{c}(2 \beta, 1)=(\sqrt{\pi} / 4) \exp \left(-\beta^{2}\right)\left\{1+2[\operatorname{erfi}(\beta / \sqrt{2})]^{2}\right\}
$$


The general expressions given in eqs (9) and (10) may be established starting from the formula given by Erdélyi [2], p. 307:

$$
\begin{aligned}
\int_{0}^{\infty} & x^{2 n} \cos (2 \beta x) \operatorname{erfc}(\alpha x) d x \\
& =\frac{\Gamma(n+1)}{\sqrt{\pi}(2 n+1) \alpha^{2 n+1}}{ }_{2} F_{2}\left(n+1, n+1 / 2 ; n+3 / 2,1 / 2 ;-\frac{\beta^{2}}{\alpha^{2}}\right) \\
& =\frac{1}{2 \alpha^{2 n+1}} \sum_{k=0}^{\infty} \frac{\Gamma(k+n+1)}{(k+n+1 / 2) \Gamma(k+1 / 2) k !}\left(-\beta^{2} / \alpha^{2}\right)^{k} .
\end{aligned}
$$

It then follows using the series expansion for $\exp \left(-x^{2}\right)$ that

$$
F_{c}(2 \beta, \alpha)=\sum_{j=0}^{\infty} \frac{(-1)^{j}}{j ! 2 \alpha^{2 j+1}} \sum_{k=0}^{\infty} \frac{\Gamma(k+j+1)}{(k+j+1 / 2) \Gamma(k+1 / 2) k !}\left(-\beta^{2} / \alpha^{2}\right)^{k} .
$$

Reversing the order of summation and employing the series representation of the hypergeometric function, it follows that

$$
F_{c}(2 \beta, \alpha)=\frac{1}{2 \alpha} \sum_{k=0}^{\infty} \frac{\left(-\beta^{2} / \alpha^{2}\right)^{k}}{\Gamma(k+3 / 2)}{ }_{2} F_{1}\left(k+1 / 2, k+1 ; k+3 / 2 ;-1 / \alpha^{2}\right) .
$$

Now, substituting the integral expression of reference [5] p. 114,

$$
{ }_{2} F_{1}\left(k+1 / 2, k+1 ; k+3 / 2 ;-1 / \alpha^{2}\right)=\frac{\Gamma(k+3 / 2)}{k ! \Gamma(1 / 2)} \int_{0}^{1} t^{k}(1-t)^{-1 / 2}\left(1+t / \alpha^{2}\right)^{-k-1 / 2} d t,
$$

and summing the geometric series, one obtains

$$
F_{c}(2 \beta, \alpha)=\frac{1}{2 \alpha \sqrt{\pi}} \int_{0}^{1} e^{-\beta^{2} t\left(\alpha^{2}+t\right)^{-1}}(1-t)^{-1 / 2}\left(1+t^{2} / \alpha^{2}\right)^{-1 / 2} d t .
$$

From this, the formula quoted in eq (10) follows immediately upon change of the integration variable from $t$ to $z$ (using the positive square root branch for $z$ ) via

$$
\left(1+t^{2} / \alpha^{2}\right)=\left(1+\alpha^{2}\right) /\left(z^{2}+\alpha^{2}\right) .
$$

The integral in eq (10), of the form

$$
T_{\alpha}(\xi) \equiv \int_{0}^{1} e^{\xi^{2} z^{2}\left(1+\alpha^{2}\right)^{-1}}\left(z^{2}+\alpha^{2}\right)^{-1} d z
$$

satisfies the differential equation

$$
\frac{d}{d \xi} e^{\xi^{2} \alpha^{2}\left(1+\alpha^{2}\right)^{-1}} T_{\alpha}(\xi)=\frac{\sqrt{\pi}}{\left(1+\alpha^{2}\right)^{1 / 2}} e^{\xi^{2} \alpha^{2}\left(1+\alpha^{2}\right)^{-1}} \operatorname{erfi}\left[\xi\left(1+\alpha^{2}\right)^{-1 / 2}\right] .
$$

Hence, integrating this equation with respect to $\xi$ over the range $(0, \beta)$, recognizing that $T_{\alpha}(0)=\alpha^{-1}$ $\arctan \left(\alpha^{-1}\right)$, and setting $\xi=\left(1+\alpha^{2}\right)^{1 / 2} x$, one finds that the expression given in eq (10) may be written in the equivalent form displayed in eq (9).

For negative $\alpha$, analogously to eq (5), the Fourier cosine transform connection is

$$
F_{c}(2 \beta,-|\alpha|)=2 F_{c}(2 \beta, 0)-F_{c}(2 \beta,|\alpha|) .
$$




\section{Evaluation of $\mathrm{I}(p, \beta, \theta ; \epsilon, \phi)$}

The five parameter definite double integral defined in eq (1) will now be evaluated in terms of the Fourier transforms discussed in the previous section. Results are quoted in eqs (15), (17), and (18). The results for the special case $\beta=0$ are given in eq (19), while the results for the limiting case $\epsilon \beta \rightarrow \gamma$ as $\beta \rightarrow 0$ are presented in eqs (2l-22).

We begin the derivation of the results by transforming the domain of the double integral using the general relation

$$
\int_{0}^{\infty} d \xi \int_{0}^{\infty} d \eta h(\xi, \eta)=\int_{0}^{\infty} \mathrm{dy} \int_{0}^{y} d x h(y-x, x),
$$

which may easily be established using the variable changes $\xi+\eta \rightarrow y, \eta \rightarrow x$. Consequently, it follows that

$$
\int_{0}^{\infty} d y \int_{0}^{y} d x h(y, x)=\int_{0}^{\infty} d y \int_{0}^{\infty} \dot{d x} h(y+x, x) .
$$

Employing this relation, we may write $I(p, \beta, \theta ; \epsilon, \phi)$ in the form

$$
\begin{aligned}
2 I(p, \beta, \theta ; \epsilon, \phi)= & \mathscr{I}_{m}\left\{\int_{0}^{\infty} d y \int_{0}^{\infty} d x e^{-p^{2}(y+x)^{2}} e^{-x^{2}} e^{i[\beta(y+x+\epsilon)+\theta+\phi]}\right. \\
& \left.+\int_{0}^{\infty} d y \int_{0}^{\infty} d x e^{-p^{2}(y+x)^{2}} e^{-x^{2}} e^{i[\beta(y+x-\epsilon)+\theta-\phi]}\right\} .
\end{aligned}
$$

At this point, $\beta$ will be restricted to values greater than zero. [The special case $\beta=0$ will be discussed later.]

In analogy with Jones and Klein [6, p. 3], we use a Dirac $\delta$-function to write the double integrals as triple integrals.

$2 I(p, \beta, \theta ; \epsilon, \phi)=$

$$
\begin{aligned}
\mathscr{I} m\left\{e^{i(\theta+\phi)} \int_{-\infty}^{\infty} d t\right. & e^{i \beta t} \int_{0}^{\infty} d y \int_{0}^{\infty} d x e^{-p^{2}(y+x)^{2}} e^{-x^{2}} \delta[t-y-(1+\epsilon) x] \\
& \left.+e^{i(\theta-\phi)} \int_{-\infty}^{\infty} d t e^{i \beta t} \int_{0}^{\infty} d y \int_{0}^{\infty} d x e^{-p^{2}(y+x)^{2}} e^{-x^{2}} \delta[t-y-(1-\epsilon) x]\right\} .
\end{aligned}
$$

Doing the integration over $y$ first and noting that $y \geq 0$ in the range of integration, we obtain

$2 I(p, \beta, \theta ; \epsilon, \phi)=$

$$
\begin{aligned}
\mathscr{I}_{m}\left\{e^{i(\theta+\phi)} \int_{-\infty}^{\infty} d t e^{i \beta t} \int_{0}^{\infty} d x e^{-p^{2}(t-\epsilon x)^{2}} e^{-x^{2}} \Theta[t-(1+\epsilon) x]\right. \\
\left.+e^{i(\theta-\phi)} \int_{-\infty}^{\infty} d t e^{i \beta t} \int_{0}^{\infty} d x e^{-p^{2}(t+\epsilon x)^{2}} e^{-x^{2}} \Theta[t-(1-\epsilon) x]\right\}
\end{aligned}
$$

where the step function $\Theta(z)$ is defined as unity for $z \geq 0$ and zero for $z<0$. The presence of the step function restricts the relevant range of integration for the variables $t$ and $x$ in the following fashion. In the first of the preceding integrals, the step function requires that $(1+\epsilon) x \leq t$, so since $x$ must be $\geq 0$, it follows that $t \geq$ 0 . Consequently, in that integral the ranges are

$$
0 \leq t<\infty \text { and } 0 \leq x \leq(1+\epsilon)^{-1} t .
$$

In the second of the preceding integrals, the step function requires that $(1-\epsilon) x \leq t$. Thus:

$$
\begin{aligned}
& \text { (i) if } \epsilon<1 \text {, then } 0 \leq x \leq(1-\epsilon)^{-1} t \text { and } 0 \leq t<\infty \text {, } \\
& \text { (ii) if } \epsilon=1 \text {, then } 0 \leq t<\infty \text { and } 0 \leq x<\infty \text {, } \\
& \text { (iii) if } \epsilon>1 \text {, then } \infty>x \geq \max \left\{-(\epsilon-1)^{-1} t, 0\right\} \text { and }-\infty<t<\infty \text {. }
\end{aligned}
$$


It is useful to define the positive quantity

$$
\mu \equiv\left(1+\epsilon^{2} p^{2}\right)^{1 / 2} .
$$

For convenience, in the first of the preceding integrals we change variables from $(t, x)$ to $(z, \xi)$ defined by

$$
z=(p / \mu) t, \quad \xi=\mu x-\epsilon p z
$$

so that the appropriate range in the first integral becomes

$$
0 \leq z<\infty, \quad-\epsilon p z \geq \xi<\left[\frac{1-\epsilon p^{2}}{(1+\epsilon) p}\right] z .
$$

Similarly, in the second of the preceding integrals, variables are changed from $(t, x)$ to $(z, \xi)$ now defined by

$$
z=(p / \mu) t, \quad \xi=\mu x+\epsilon p z
$$

so that appropriate ranges for the second integral for the various cases becomes

(i) if $\epsilon<1$, then $0 \leq z<\infty, \epsilon p z \leq \xi \leq\left[\frac{1+\epsilon p^{2}}{(1-\epsilon) p}\right] z$,

(ii) if $\epsilon=1$, then $0 \leq z<\infty$, epz $\leq \xi<\infty$,

(iii) if $\epsilon>1$, then $-\infty<z<\infty, \max \left\{\frac{-\left(1+\epsilon p^{2}\right)}{(\epsilon-1) p} z, \epsilon p z\right\} \leq \xi<\infty$.

Making these substitutions in the preceding integrals we find

$2 p I(p, \beta, \theta ; \epsilon, \phi)=$

$$
\begin{aligned}
\mathscr{I} m\left\{e^{i(\theta+\phi)} \int_{0}^{\infty} d z e^{i(\beta \mu / p) z} e^{-z^{2}} \int_{-\epsilon p z}^{\left[\frac{1-\epsilon p^{2}}{(1+\epsilon) p}\right]} d \xi e^{-\xi^{2}}\right. & \\
& \left.+e^{i(\theta-\phi)} \int_{E}^{\infty} d z e^{i(\beta \mu / p) z} e^{-z^{2}} \int_{A(z)}^{B(z)} d \xi e^{-\xi^{2}}\right\}
\end{aligned}
$$

where

(i) if $\epsilon<1$, then $E=0, A(z)=\epsilon p z, B(z)=\left[\frac{1+\epsilon p^{2}}{(1-\epsilon) p}\right] z$,

(ii) if $\epsilon=1$, then $E=0, A(z)=\epsilon p z, B(z)=\infty$,

(iii) if $\epsilon>1$, then $E=-\infty, A(z)=\max \left\{\frac{-\left(1+\epsilon p^{2}\right)}{(\epsilon-1) p} z, \epsilon p z\right\}, B(z)=\infty$.

From the definition of the erfc function and eq (4), it follows that

(i) for $0 \leq \epsilon<1, \quad \mu=\left(1+\epsilon^{2} p^{2}\right)^{1 / 2}$ :

$$
\begin{aligned}
& 4 \pi^{-1 / 2} p I(p, \beta, \theta ; \epsilon, \phi) \\
& =\mathscr{I}_{m}\left\{e^{i(\theta+\phi)} \int_{0}^{\infty} d z e^{i(\beta \mu / p) z} e^{-z^{2}}\left[2 \operatorname{erfc}(0)-\operatorname{erfc}(\epsilon p z)-\operatorname{erfc}\left(\frac{\left(1-\epsilon p^{2}\right.}{(1+\epsilon) p} z\right)\right]\right. \\
& \left.+e^{i(\theta-\phi)} \int_{0}^{\infty} d z e^{i(\beta \mu / p) z} e^{-z^{2}}\left[\operatorname{erfc}(\epsilon p z)-\operatorname{erfc}\left(\frac{\left(1+\epsilon p^{2}\right)}{(1-\epsilon) p} z\right)\right]\right\} ;
\end{aligned}
$$


(ii) for $\epsilon=1, \quad \mu=\left(1+p^{2}\right)^{1 / 2}$ :

$$
\begin{aligned}
& 4 \pi^{-1 / 2} p I(p, \beta, \theta ; 1, \phi) \\
& \quad=\operatorname{Im}\left\{\left\{e^{i(\theta+\phi)} \int_{0}^{\infty} d z e^{i(\beta \mu / p) z} e^{-z^{2}}\left[2 \operatorname{erfc}(0)-\operatorname{erfc}(p z)-\operatorname{erfc}\left(\frac{\left.1-p^{2}\right)}{2 p} z\right)\right]\right.\right. \\
& \left.+e^{i(\theta-\phi)} \int_{0}^{\infty} d z e^{i(\beta \mu / p) z} e^{-z^{2}} \operatorname{erfc}(p z)\right\} ;
\end{aligned}
$$

(iii) for $\epsilon>1, \quad \mu=\left(1+\epsilon^{2} p^{2}\right)^{1 / 2}$ :

$$
\begin{aligned}
& 4 \pi^{-1 / 2} p I(p, \beta, \theta ; \epsilon, \phi) \\
& \quad \mathscr{I m}\left\{e^{i(\theta+\phi)} \int_{0}^{\infty} d z e^{i(\beta \mu / p) z} e^{-z^{2}}\left[2 \operatorname{erfc}(0)-\operatorname{erfc}(\epsilon p z)-\operatorname{erfc}\left(\frac{\left(1-\epsilon p^{2}\right)}{(1+\epsilon) p} z\right)\right]\right. \\
& +e^{i(\theta-\phi)} \int_{0}^{\infty} d z e^{-i(\beta \mu / p) z} e^{-z^{2}} \operatorname{erfc}\left(\frac{\left(1+\epsilon p^{2}\right)}{(\epsilon-1) p} z\right) \\
& \left.+e^{i(\theta-\phi)} \int_{0}^{\infty} d z e^{i(\beta \mu / p) z} e^{-z^{2}} \operatorname{erfc}(\epsilon p z)\right\} .
\end{aligned}
$$

Using the definitions of the Fourier transforms $F_{c}$ and $F_{s}$ given in the previous section, these results may be put in the form:

(i) for $0 \leq \epsilon<1, \quad \mu=\left(1+\epsilon^{2} p^{2}\right)^{1 / 2}$;

$$
\begin{aligned}
4 \pi^{-1 / 2} p I(p, \beta, \theta ; \epsilon, \phi) \\
\quad=\sin (\theta+\phi)\left[2 F_{c}(\beta \mu / p, 0)-F_{c}\left(\beta \mu / p, \frac{1-\epsilon p^{2}}{(1+\epsilon) p}\right)\right] \\
+\cos (\theta+\phi)\left[2 F_{s}(\beta \mu / p, 0)-F_{s}\left(\beta \mu / p, \frac{1-\epsilon p^{2}}{(1+\epsilon) p}\right)\right] \\
\quad-\sin (\theta-\phi) F_{c}\left(\beta \mu / p, \frac{1+\epsilon p^{2}}{(1-\epsilon) p}\right)-\cos (\theta-\phi) F_{s}\left(\beta \mu / p, \frac{1+\epsilon p^{2}}{(1-\epsilon) p}\right) \\
+2 \sin (\phi)\left[-\cos (\theta) F_{c}(\beta \mu / p, \epsilon p)+\sin (\theta) F_{s}(\beta \mu / p, \epsilon p)\right] .
\end{aligned}
$$

[Note: The Fourier transforms have been defined for non-negative values of their second arguments. When $\left(1-\epsilon p^{2}\right)<0$, the above expressions may be used by replacing

$$
2 F(\beta \mu / p, 0)-F\left(\beta \mu / p, \frac{1-\epsilon p^{2}}{(1+\epsilon) p}\right)=F\left(\beta \mu / p, \frac{\epsilon p^{2}-1}{(1+\epsilon) p}\right),
$$

where $F$ represents either the cosine or the sine transform. This replacement follows from eqs (5) and (12).]

(ii) for $\epsilon=1, \quad \mu=\left(1+p^{2}\right)^{1 / 2}$;

$$
\begin{aligned}
& 4 \pi^{-1 / 2} p I(p, \beta, \theta ; 1, \phi) \\
& \quad=\sin (\theta+\phi)\left[2 F_{c}(\beta \mu / p, 0)-F_{c}\left(\beta \mu / p, \frac{1-p^{2}}{2 p}\right)\right] \\
& +\cos (\theta+\phi)\left[2 F_{s}(\beta \mu / p, 0)-F_{s}\left(\beta \mu / p, \frac{1-p^{2}}{2 p}\right)\right] \\
& \quad+2 \sin (\phi)\left[-\cos (\theta) F_{c}(\beta \mu / p, p)+\sin (\theta) F_{s}(\beta \mu / p, p)\right] .
\end{aligned}
$$


Again, for $1-p^{2}<0$, the above expressions may be used if we make the replacement given in eq (16) with $\epsilon=1$.

$$
\begin{aligned}
& \text { (iii) for } \quad \epsilon>1, \quad \mu=\left(1+\epsilon^{2} p^{2}\right)^{1 / 2} ; \\
& 4 \pi^{-1 / 2} p I(p, \beta, \theta ; \epsilon, \phi) \\
& \quad=\sin (\theta+\phi)\left[2 F_{c}(\beta \mu / p, 0)-F_{c}\left(\beta \mu / p, \frac{1-\epsilon p^{2}}{(1+\epsilon) p}\right)\right] \\
& +\cos (\theta+\phi)\left[2 F_{s}(\beta \mu / p, 0)-F_{s}\left(\beta \mu / p, \frac{1-\epsilon p^{2}}{(1+\epsilon) p}\right)\right] \\
& +\sin (\theta-\phi) F_{c}\left(\beta \mu / p, \frac{1+\epsilon p^{2}}{(\epsilon-1) p}\right)-\cos (\theta-\phi) F_{s}\left(\beta \mu / p, \frac{1+\epsilon p^{2}}{(\epsilon-1) p}\right) \\
& +2 \sin (\phi)\left[-\cos (\theta) F_{c}(\beta \mu / p, \epsilon p)+\sin (\theta) F_{s}(\beta \mu / p, \epsilon p)\right] .
\end{aligned}
$$

Here, when $1-\epsilon \mathrm{p}^{2}<0$, the replacement given in eq (16) should be made.

The various results given for $I(p, \beta, \theta ; \epsilon \phi)$ are continuous for $\epsilon=1$. Using the fact that $F_{c}(\beta, x) \rightarrow 0$ and $F_{s}(\beta, \mathrm{x}) \rightarrow 0$ as $x \rightarrow \infty$, we may readily verify that

$$
\lim _{\epsilon \rightarrow 1^{-}} I(p, \beta, \theta ; \epsilon, \phi)=I(p, \beta, \theta ; 1, \phi)
$$

and

$$
\lim _{\epsilon \rightarrow 1^{+}} I(p, \beta, \theta ; \epsilon, \phi)=I(p, \beta, \theta ; 1, \phi) .
$$

For the special case $\beta=0$, one obtains directly from the definition of the double integral under discussion,

$$
\begin{aligned}
I(p, 0, \theta ; \boldsymbol{\epsilon}, \phi) & =\int_{0}^{\infty} d y \int_{0}^{y} d x \exp \left(-p^{2} y^{2}\right) \exp \left(-x^{2}\right) \sin \theta \sin \phi \\
& =\sin \theta \cos \phi \int_{0}^{\infty} d y \exp \left(-p^{2} y^{2}\right)[\operatorname{erfc}(0)-\operatorname{erfc}(y)] \\
& =\sqrt{\pi}(2 p)^{-1} \sin \theta \cos \phi\left[F_{c}(0,0)-F_{c}(0,1 / p)\right]
\end{aligned}
$$

Thus, it follows using the specific values of the transforms,

$$
\begin{aligned}
I(p, 0, \theta ; \epsilon, \phi) & =(4 p)^{-1} \sin \theta \cos \phi[\pi-2 \arctan (p)] \\
& =(2 p)^{-1} \sin \theta \cos \phi \arctan (1 / p) .
\end{aligned}
$$

This result also follows by a limiting process $\beta \rightarrow 0$ in the results quoted in eqs (15-18) upon application of the relation for positive arguments

$$
\arctan (\mathrm{x})+\arctan (1 / x)=\pi / 2,
$$

and the identities (for $\epsilon \geq 0, p>0$ ):

$$
\arctan (\epsilon p) \pm \arctan (p)= \pm \arctan \left[\frac{(1 \pm \epsilon) p}{1 \mp p^{2}}\right] \text { for } \quad 1-\epsilon p^{2}>0,
$$

and

$$
\arctan \left(\frac{1}{p}\right) \pm \arctan \left(\frac{1}{\epsilon p}\right)= \pm \arctan \left[\frac{(1 \pm \epsilon) p}{\epsilon p^{2} \mp 1}\right] \text { for } \epsilon p^{2}-1>0
$$


[The inequivalent forms corresponding to the two upper signs are necessitated by the arctangent's definition which requires $\pi / 2 \leq \arctan (\mathrm{x}) \leq \pi / 2$.]

Finally, the particular double integral

$$
H(\gamma, p, \phi) \equiv \int_{0}^{\infty} d y \int_{0}^{y} d x \exp \left(-p^{2} y^{2}\right) \exp \left(-x^{2}\right) \cos (\gamma \mathrm{x}+\phi), \gamma \geq 0
$$

may most easily be evaluated by integration by parts:

$$
\begin{aligned}
H(\gamma, p, \phi) & =-(1 / p) \int_{y=0}^{\infty}[\operatorname{derfc}(y)] \int_{x=0}^{y / p} d x \exp \left(-x^{2}\right) \cos (\gamma x+\phi) \\
& =\sqrt{\pi}(2 p)^{-1}\left[(\cos \phi) F_{c}(\gamma, p)-(\sin \phi) F_{s}(\gamma, p)\right] .
\end{aligned}
$$

This result also follows directly from Eq (18) by the following limit process:

$$
H(\gamma, p, \phi)=\lim _{\beta \rightarrow 0^{+}} I(p, \beta, \pi / 2 ; \gamma / \beta, \phi) .
$$

The author would like to thank Professor N. Kemmer for his hospitality at the University of Edinburgh where this work was initiated during a sabbatical leave.

\section{References}

[1] Ng, E. W. and Geller, M., A Table of Integrals of the Error Functions, J. Res. Nat. Bur. Stand. (U.S.) 73B (Math. Sci.), No. 1, 1-20 (Jan.-Mar. 1969).

[2] Erdéyli, A., Magnus, W., Oberhettinger, F., and Tricomi, F. G., Table of Integral Transforms, Vol. 2 (McGraw-Hill Book Co., Inc., New York 1954).

[3] Rosser, J. B., The Theory and Application of $\int_{0}^{z} \exp \left(-x^{2}\right) d x$ and $\int_{0}^{z} \exp \left(-p^{2} y^{2}\right) d y \int_{0}^{y} \exp \left(-x^{2}\right) d x$, (Mapleton House, Brooklyn, N.Y. 1948).

[4] Ng, E. W. and Geller, M., A Table of Integrals of the Error Function. II. Additions and Corrections, J. Res. Nat. Bur. Stand. (U.S.) 75B (Math. Sci.), Nos. 3 and 4, 149-163 (1971).

[5] Erdéyli, A., Magnus, W., Oberhettinger, F., and Tricomi, F. G., Higher Transcendental Functions, Vol. 1 (McGraw Hill Book Co., Inc., New York 1953).

[6] Jones, D. S. and Klein, M., Asymptotic Expansion of Multiple Integrals and the Method of Stationary Phase, J. Math. and Physics (MIT), 37, 1-28 (1958). 